\title{
Fourth-Order Bandpass Filter Using Dual-Mode Ring Resonator and Parallel-Coupled Lines
}

\author{
Zekai $\mathrm{Luo}^{1}$, Kai Da Xu ${ }^{1,2,{ }^{*}}$, Yijun $\mathrm{Cai}^{3}$ and Yanhui $\mathrm{Liu}^{1}$ \\ ${ }^{1}$ Institute of Electromagnetics and Acoustics \& Department of Electronic Science, Xiamen University, Xiamen 361005, China \\ ${ }^{2}$ Shenzhen Research Institute of Xiamen University, Shenzhen 518057, China \\ ${ }^{3}$ School of Optoelectronic and Communication Engineering, Xiamen University of Technology, Xiamen 361024, China
}

*kaidaxu@xmu.edu.cn

\begin{abstract}
A fourth-order bandpass filter using dual-mode ring resonator and parallel-coupled lines loaded with half-wavelength open stubs is presented in this paper. Six transmission zeros (TZs) and four transmission poles (TPs) from 0 to $2 f_{0}\left(f_{0}\right.$ denotes center frequency of the filter passband) can be achieved with the help of two open stubs and open coupled lines, where two TZs are generated by the loaded two open stubs. Finally, a verified physical layout of the proposed filter is designed and simulated with good frequency selectivity, whose ADS and HFSS simulations are in good agreement.
\end{abstract}

\section{INTRODUCTION}

Microwave wideband bandpass filters (BPFs) with high frequency selectivity have been gaining much attention in the modern wireless communication systems [1-3]. Therefore, a variety of approaches have been proposed by microwave researchers and engineers recently to design high-performance BPFs. In [4], a seventh-order wideband BPF using coupled lines and open/shorted stubs was designed, whose frequency selectivity and return loss become better when open and shorted stubs are loaded near the input and output ports. In [56], a dual-mode ring resonator was introduced and utilized to construct high-selectivity BPFs, where the resonator was fed by quarter-wavelength side-coupled lines to fix transmission zeros frequencies.

In this paper, a novel high selectivity fourth-order BPF using dual-mode resonator and parallel-coupled lines with loaded open stubs is proposed and analyzed. With this simple design, four transmission poles (TPs) and six transmission zeros (TZs) can be realized. The BPF prototype centering at 2.0 $\mathrm{GHz}$ is constructed on a dielectric substrate with the relative permittivity of 2.65 and thickness of $1 \mathrm{~mm}$.

\section{Filter Design AND ANALYSIS}

The ideal circuit of the proposed BPF using dual-mode ring resonator and parallel-coupled lines is shown in Fig. 1. The dual-mode ring resonator located in the middle of the circuit is fed by two quarter-wavelength side-coupled lines, whose even and odd mode characteristic impedances are $Z_{0 \mathrm{e} 1}$ and $Z_{0 \mathrm{ol}}$, respectively, and electrical length is $\theta$. Two open coupled lines ( $Z_{0 \mathrm{e} 2}, Z_{0 \mathrm{o}}$, electrical length $\theta$ ) with two open loaded stubs $\left(Z_{2}\right.$, electrical length $2 \theta$ ) are located close to the input and output ports. Accordingly, the simulated $S$-parameters of this ideal filter circuit using ADS software is illustrated in Fig. 2. High frequency selectivity with four TPs and six TZs can be achieved. When two half-wavelength open stubs are shunted near the input and output ports, two more TZs $\left(f_{\mathrm{tz} 2}\right.$ and $\left.f_{\mathrm{tz} 5}\right)$ are generated at $0.5 f_{0}$ and $1.5 f_{0}$ compared with those of filter circuit without open stubs, as seen in Fig. 2. The positions of four TPs and the other four TZs $\left(f_{\mathrm{tz} 1}, f_{\mathrm{tz} 3}, f_{\mathrm{tz} 4}\right.$, and $\left.f_{\mathrm{tz} 6}\right)$ remain unchanged.

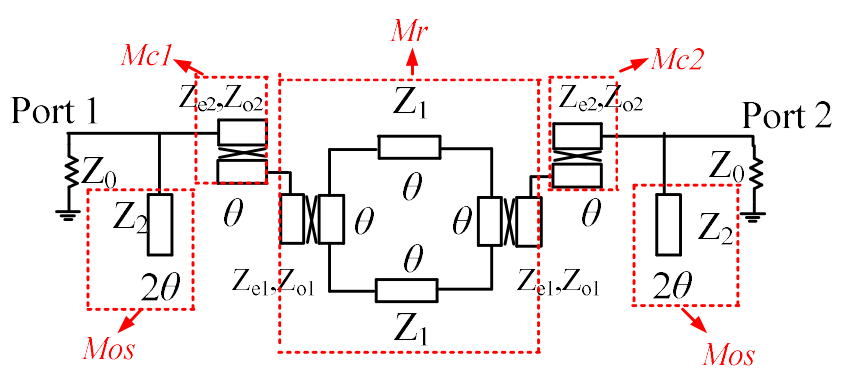

Fig. 1. Ideal circuit of the proposed filter

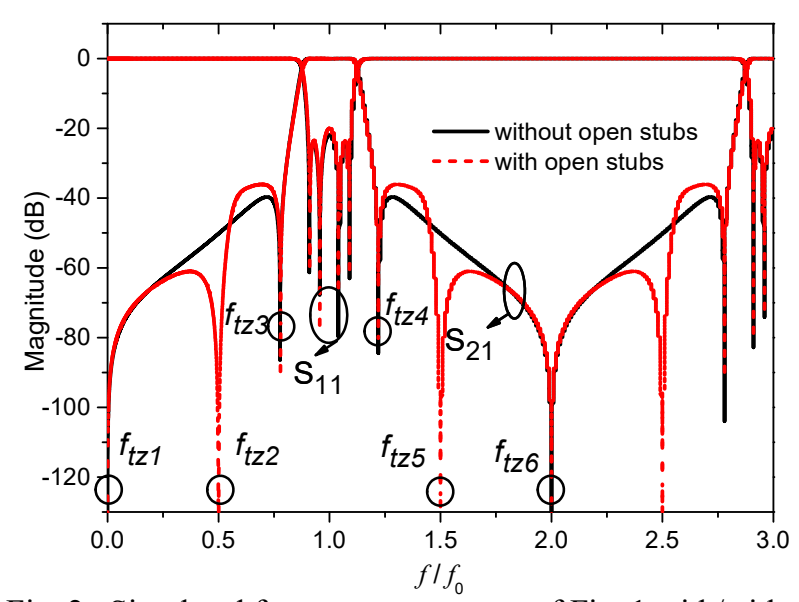

Fig. 2. Simulated frequency responses of Fig. 1 with/without open stubs $\left(Z_{1}=93 \Omega, Z_{2}=186 \Omega, Z_{\mathrm{e} 1}=160 \Omega, Z_{\mathrm{o} 1}=77 \Omega\right.$, $\mathrm{Z}_{\mathrm{e} 2}=129 \Omega, \mathrm{Z}_{\mathrm{o} 2}=57 \Omega$, and $\theta=90^{\circ}$ )

The $A B C D$ matrix of the filter circuit in Fig. 1 can be defined as $M_{o s} \times M_{c 1} \times M_{r} \times M_{c 2} \times M_{o s}$, where $M_{o s}$ is the $A B C D$ matrix of the loaded half-wavelength open stub, $M_{c 1}$ and $M_{c 2}$ are the $A B C D$ matrices of the open coupled lines 
obtained from [7], and the $A B C D$ matrix $M r$ can be directly deduced from [5]. Simply, the $A B C D$ matrix of $M_{o s}$ is:

$$
A B C D_{M_{\mathrm{os}}}=\left[\begin{array}{ll}
1 & 0 \\
Y & 1
\end{array}\right]
$$

where $Y=\frac{j \tan 2 \theta}{Z_{2}}$.

After $A B C D$-, $Y$ - and $S$-parameter conversions, we can obtain the $S$-matrix of the whole circuit in Fig. 1. The locations of transmission zeros can be determined when $S_{21}=0$. Through calculation, six transmission zeros can be obtained, which are expressed as:

$$
\begin{aligned}
& \theta_{t z 1}=0, \quad \theta_{t z 6}=\pi \\
& \theta_{t z 2}=\pi / 4, \quad \theta_{t z 5}=3 \pi / 4 \\
& \theta_{t z 3}=\arccos \sqrt{\frac{Z_{e 1}+Z_{o 1}-2 Z_{1}}{Z_{e 1}+Z_{o 1}+2 Z_{1}}}, \quad \theta_{t z}=\pi-\theta_{t z 3}
\end{aligned}
$$

where $\theta_{t z 2}$ and $\theta_{t z 5}$ corresponding to the two TZs $f_{\mathrm{tz} 2}=0.5 f_{0}$ and $f_{\mathrm{tz} 5}=1.5 f_{0}$, respectively, are yielded by the loaded halfwavelength open stubs.

Fig. 3 illustrates a verified physical layout of the proposed filter designed on the dielectric substrate with the relative permittivity of 2.65 and thickness of $1 \mathrm{~mm}$. From Fig. 4 , we can see that the simulated responses of ADS and HFSS including insertion losses and return losses are in good agreement.

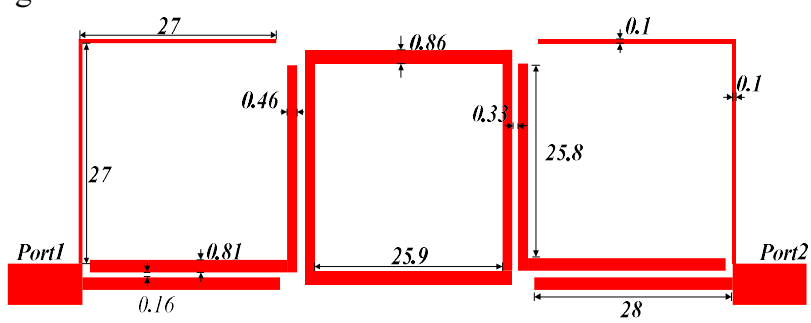

Fig. 3. Layout of the proposed BPF (not to scale, unit: $\mathrm{mm}$ )

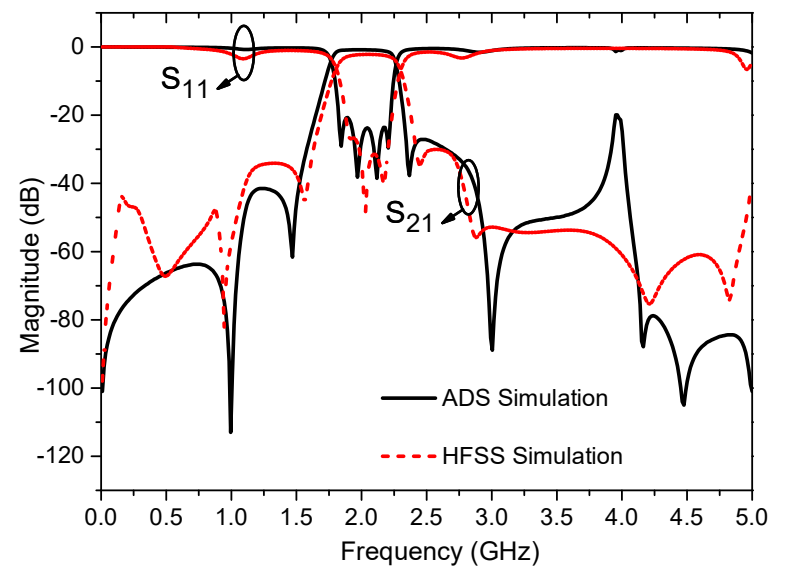

Fig. 4. Simulated frequency responses of the proposed BPF.

\section{CONCLUSION}

This paper presents a high-selectivity bandpass filter using dual-mode ring resonator and parallel-coupled lines loaded with half-wavelength open stubs. Its six TZs can be deduced through ABCD matrix calculation, which can be found that two of them are generated by the loaded half-wavelength open stubs. Due to its simple structure, the proposed filter layout can be also extended to the design of balanced filter circuits.

\section{ACKNOWLEDGMENT}

This work was supported in part by the National Natural Science Foundation of China (No. 61601390), and Shenzhen Science and Technology Innovation Project (No. JCYJ20170306141249935).

\section{REFERENCES}

[1] S. Sun and L. Zhu, "Multimode-resonator-based bandpass filters," IEEE Microw. Mag., vol. 10, no. 2, pp. 88-98, Apr. 2009.

[2] W. J. Feng, W. Q. Che, and Q. Xue, "Transversal signal interaction: Overview of high-performance wideband bandpass filters," IEEE Microw. Mag., vol. 15, no. 2, pp. 84-96, Mar. 2014.

[3] J. S. Hong and M. J. Lancaster, Microstrip Filters for RF/Microwave Applictions. New York: Wiley, 2001.

[4] K. D. Xu, F. Y. Zhang, Y. H. Liu, and W. Nie, "High selectivity seventh-order wideband bandpass filter using coupled lines and open/shorted stubs," Electron. Lett., 2017, DOI: 10.1049/el.2017.4233.

[5] M. Salleh, G. Prigent, O. Pigaglio, and R. Crampagne, "Quarterwavelength side-coupled ring resonator for bandpass filters," IEEE Trans. Microw. Theory Technol., vol. 56, no. 1, pp. 156-162, Jan. 2008.

[6] W. J. Feng, X. Gao, W. Q. Che, and Q. Xue, "Bandpass filter loaded with open stubs using dual-mode ring resonator," IEEE Microw. Wireless Compon Lett., vol. 25, no. 5, pp. 295-297, May 2015.

[7] G. Matthaei, L. Young, and E. M. T. Jones, Microwave Filters, Impedance Matching Networks and Coupling Structures. Norwood, MA, USA: Artech House, 1985, chapter 5, sec. 5, pp. 221. 